\title{
Ligustilide alleviates podocyte injury via suppressing the SIRT1/ NF- $\kappa B$ signaling pathways in rats with diabetic nephropathy
}

\author{
Feng Xu ${ }^{1,2}$, Xingxing Fang ${ }^{3}, \mathrm{Zi} \mathrm{Ye}^{4}$, Shuo Tao ${ }^{3}$, Wangshu Liu ${ }^{2}$, Jianbing Su${ }^{1}$, Xueqin Wang ${ }^{1}$ \\ ${ }^{1}$ Department of Endocrinology, the Affiliated Hospital 2 of Nantong University and First People's Hospital of Nantong City, Nantong, China; \\ ${ }^{2}$ Department of Endocrinology, Second Affiliated Hospital of Soochow University, Suzhou, China; ${ }^{3}$ Department of Nephrology, ${ }^{4}$ Department of \\ Neurosurgery, the Affiliated Hospital 2 of Nantong University and First People's Hospital of Nantong City, Nantong, China \\ Contributions: (I) Conception and design: F Xu; (II) Administrative support: Z Ye; (III) Provision of study materials or patients: X Fang; (IV) \\ Collection and assembly of data: S Tao; (V) Data analysis and interpretation: X Wang; (VI) Manuscript writing: All authors; (VII) Final approval of \\ manuscript: All authors. \\ Correspondence to: Xingxing Fang; Zi Ye. Department of Endocrinology, the First People's Hospital of Nantong, Nantong, China. \\ Email: xufeng5205529@163.com.
}

Background: Diabetic nephropathy (DN) is one of the common chronic microvascular complications of diabetes, and podocyte injury and dysfunction are strictly related to the pathogenesis of DN. Studies have shown that ligustilide (LIG) has anti-inflammatory, antioxidant, and anti-apoptotic activities. This study was designed to investigate the therapeutic effect of LIG in DN rats and their mechanisms.

Methods: DN rat models ( $\mathrm{n}=10$ ) were induced by streptozotocin (STZ) combined with a high-fat diet. Rats in the LIG group were intragastrically administered with LIG daily for eight weeks, and animals in the positive control group were treated with Losartan potassium. The body weight and blood glucose were checked weekly during the treatment. The pathological changes of kidney tissue were observed with hematoxylin and eosin $(\mathrm{HE})$ staining. Blood lipid profiles and renal function-related markers, including total cholesterol (TC), triglyceride (TG), low-density lipoprotein cholesterol (LDL-C), high-density lipoprotein cholesterol (HDL-C), blood urea nitrogen (BUN), and serum creatinine (Scr) were monitored using a biochemical analyzer. The protein expression of nephrin was determined by immunohistochemistry and Western blotting. Finally, Western blot was used to determine the protein expression of Sirtuin 1 (SIRT1) and nuclear factor-kappa B (NF-кB).

Results: Compared with the healthy control group, rats in the DN group have slower weight gain, increased blood sugar level, renal lesions, and impaired renal function, along with decreased nephrin expression, abnormally activated NF- $\mathrm{KB}$, and inhibited SIRT1 protein expression. All the above conditions were improved after intervention with either losartan potassium or LIG.

Conclusions: LIG attenuates podocyte injury by regulating the SIRT1/NF- $\kappa \mathrm{B}$ signaling pathway and thereby exerts its protective effect on renal function in DN rats.

Keywords: Ligustilide (LIG); podocyte injury; Sirtuin 1/nuclear factor-kappa B signaling pathway (SIRT1/NF- $\mathrm{B}$ signaling pathway); diabetic nephropathy (DN)

Submitted Jul 07, 2020. Accepted for publication Sep 16, 2020.

doi: 10.21037/atm-20-5811

View this article at: http://dx.doi.org/10.21037/atm-20-5811

\section{Introduction}

Diabetic nephropathy (DN) is one of the common chronic microvascular complications of diabetes. Microalbuminuria features early DN; as the disease progresses, a substantial amount of proteinuria occurs, along with elevated serum creatinine (Scr) and urea nitrogen levels. Finally, the patients will develop the end-stage renal disease (ESRD) $(1,2)$. It has been found that podocyte injury and dysfunction are related to the pathogenesis of DN (3). Podocytes are terminally differentiated epithelial cells that have three 
segments: the cell body, significant processes, and foot processes. A slit diaphragm connects foot processes, the final barrier in the glomerular filtration barrier. When this barrier is damaged, the podocyte foot process is extensively fused, the slit diaphragm disappears, the glomerular filtration rate decreases and proteinuria occurs (4). The injured podocytes may undergo epithelial-to-mesenchymal transition (EMT) by down-regulating the expression of a series of epithelial markers (including nephrin) (5). Studies have shown that a high-glycemic environment can promote the development of podocyte EMT (6), and the oxidative stress caused by hyperglycemia is a contributor to podocyte injury in the course of DN (7). Therefore, research on the injury mechanism of podocytes offers a new direction for the clinical treatment of DN.

Ligustilide (LIG) is a typical phthalide compound that accounts for the main pharmaceutical activities in traditional Chinese drugs, including Radix Angelicae Sinensis, Rhizoma Ligustici Cbuanxiong, and other plants in the family Apiaceae (8). Due to its unsaturated phthalide structure and butenyl, LIG can easily experience various reactions, including dehydrogenation, oxidation, and isomerization. According to literature, LIG has anti-inflammatory (9), antioxidant (10,11), anti-apoptosis (12), and many other pharmacological activities. Here, we investigated the potential protective effect of LIG on podocyte injury in DN and explored its mechanisms.

We present the following article in accordance with the ARRIVE reporting checklist (available at http://dx.doi. org/10.21037/atm-20-5811).

\section{Methods}

\section{Reagents and equipment}

Streptozotocin (STZ) was bought from Sigma-Aldrich, USA. Protein lysate was bought from Invitrogen, USA. Rabbit polyclonal anti-nephrin antibody (No. ABT331) was bought from Sigma-Aldrich, USA. A chromogenic kit for Western blotting was bought from Millipore Corporation (USA). The PVDF membrane was bought from Millipore (Billerica, MA, USA). ELISA kit was bought from Uscn Life Science Inc., USA. EPS-300 electrophoresis tank was bought from Tanon, Shanghai, China. Tanon 5200 chemiluminescence imaging analysis system was bought from Tanon, Shanghai, China. The cryostat was bought from Leica Biosystems, Germany. The inverted microscope was bought from Carl Zeiss, Germany.

\section{Experimental animals}

SPF-grade male SD rats, aged five weeks and weighing $190.0 \pm 2.0 \mathrm{~g}$, were purchased from the Center of Laboratory Animals, Nantong University [license number: SCXK (Su)2019-0003] and housed at the animal room of Nantong University. The office of the Laboratory Animal Welfare and the Ethics Committee at Nantong University approved the study. The breeding of these animals followed the requirements in the Regulations of the People's Republic of China on the Management of Laboratory Animals and the Measures for the Quality Management of Laboratory Animals (Animal Ethics Review Number: No. S20191021-003). The relative humidity of the animal breeding environment was $55-65 \%$, and the relative temperature was $22-24$ degrees Celsius.

\section{Creation of DN rat models and drug administration}

Fifty rats were acclimatized to the laboratory for three days. Rats in the normal group were fed a normal healthy rat diet, whereas animals in the modeling group were given a high-fat diet [MD12033 (60\% Kcal fat), purchased from Jiangsu Medicience Biomedicine Co., Ltd., China] for four weeks. After five weeks, rats in the modeling group were intraperitoneally injected with $1 \%$ STZ (Sigma-Aldrich, St. Louis, MO, USA) solution [freshly prepared in sodium citrate buffer $(0.1 \mathrm{~mol} / \mathrm{L}, \mathrm{pH} 4.5)$, stored in the dark, and sterilized by using a $0.2-\mu \mathrm{m}$ microporous membrane] at a dose of $35 \mathrm{mg} / \mathrm{kg}$. The normal group was injected with an equal volume of sodium citrate buffer $(0.1 \mathrm{~mol} / \mathrm{L}, \mathrm{pH} 4.5)$. After 72 hours, the blood sample was taken from the tail vein to measure the blood glucose level after a 12-hour fast, and a fasting blood glucose level of $\geq 16.7 \mathrm{mmol} / \mathrm{L}$ indicated successful modeling of DN rats. Rats that failed in the model establishment were sacrificed. Diabetic rats were randomly divided into four groups: $\mathrm{DN}$ group $(\mathrm{n}=10)$, positive control group $(\mathrm{n}=10$; administered with Losartan potassium), low-dose LIG group ( $\mathrm{n}=10$; administered with $20 \mathrm{mg} / \mathrm{d}$ LIG) (12), and high-dose LIG group ( $\mathrm{n}=10$; administered with $80 \mathrm{mg} / \mathrm{d}$ LIG) (12). LIG was intragastrically administered for six weeks. Body weight was measured once weekly, and the water intake was recorded. Twenty-four-hour urine samples were collected, and the urine output was recorded weekly.

Upon the completion of the intragastric administration of the drug(s), rats were anesthetized with $10 \%$ chloral hydrate solution; the chest was opened, and blood samples 
were harvested from the heart and then stored in a standard serum tube. The kidneys were harvested and weighed after their capsules were removed. They were cut open along their long axis. The right kidney was fixed with $4 \%$ paraformaldehyde before it was prepared into paraffinembedded sections for histopathology and frozen sections for immunohistochemical detection. For the left kidney, after its capsule was stripped away, longitudinal dissection was performed towards the cortex, which was cut into pieces, placed in a sterile cryotube, immediately put into liquid nitrogen for quick freezing, and finally quickly transferred and stored in a refrigerator at $-80^{\circ} \mathrm{C}$ for the subsequent Western blotting.

\section{Glucose tolerance test (GTT) and insulin tolerance test (ITT)}

GTT: rats were fasted beginning at $5 \mathrm{PM}$ on the day before the experiment and were allowed to drink water after $9 \mathrm{AM}$ the next morning. Glucose solution is intraperitoneally injected at a dose of $2 \mathrm{~g} / \mathrm{kg}$. Blood glucose level was measured before injection in each rat with an interval of $1 \mathrm{~min}$. Furthermore, blood glucose levels were measured at time points of 15, 30, 60, 90, and $120 \mathrm{~min}$ after glucose injection by an electronic glucometer using blood samples collected from the tail vein.

ITT: rats fasted for four hours. The insulin solution was intraperitoneally injected at a dose of $1 \mathrm{U} / \mathrm{kg}$. Blood glucose level was measured before injection in each rat with an interval of $1 \mathrm{~min}$. Furthermore, blood glucose levels were measured at time points of $15,30,60$, and $90 \mathrm{~min}$ after insulin injection by an electronic glucometer using blood samples collected from the tail vein.

\section{The pathological examination}

The rat kidney tissue was fixed with $4 \%$ paraformaldehyde at room temperature for $24 \mathrm{~h}$ and embedded in paraffin. The tissue was cut into $4-\mu \mathrm{m}$ sections for deparaffinization, hematoxylin staining, hydrochloric acid differentiation, and eosin staining for $1 \mathrm{~min}$. The resulting block was dehydrated and cleared through graded ethanol, sealed with neutral gum, and then placed on a laser confocal microscope $(\times 200)$ to observe the pathological changes of the renal tissue.

\section{The biochemical tests}

Blood lipid profiles and renal function-related markers include total cholesterol (TC), triglyceride (TG), low- density lipoprotein cholesterol (LDL-C), high-density lipoprotein cholesterol (HDL-C), blood urea nitrogen (BUN), and Scr were monitored using an automatic biochemical analyzer (kits provided by Daiichi Chemical Industry Co., Japan; Olympus 1000).

\section{Immunobistochemical examinations}

The sacrificed rats were fixed with $4 \%$ paraformaldehyde. The kidney tissue was harvested en bloc and fixed in 4\% paraformaldehyde solution overnight, dehydrated serially in sucrose solutions, and cut into $50-\mu \mathrm{m}$ sections using a cryostat (CM1950, Leica Biosystems, Nussloch, Germany). The sections were blocked with $1 \%$ normal fetal goat serum for $60 \mathrm{~min}$ and then incubated in rabbit anti-nephrin polyclonal antibody (1:500, No. ABT331, Sigma-Aldrich) at $4{ }^{\circ} \mathrm{C}$ overnight, followed by incubation with the secondary antibody at $37^{\circ} \mathrm{C}$ for 1 hour and DAB staining. The optical density of nephrin, which reflects its immunoreactivity, was measured using ImageJ software. The average of the values obtained from every five animals was taken as the average of each group.

\section{Western blotting}

After treatment with pre-cooled RIPA (Invitrogen, Carlsbad, California, USA), the kidney tissue was homogenized and lysed on ice for 30 minutes to extract the total protein. A protein sample $(25 \mu \mathrm{G})$ was added into a $10 \%$ SDS-polyacrylamide gel electrophoresis (SDSPAGE). After electrophoresis, the proteins are transferred with the membrane. The PVDF membrane was transferred to $5 \%$ skim milk powder dissolved in TBST and blocked at room temperature for two hours. The membranes were then incubated overnight at $4{ }^{\circ} \mathrm{C}$ with each diluted primary antibody. After washing with a TBST solution three times, the horseradish peroxidase-labeled second antibodies were added, followed by one hour of incubation at room temperature. After rinsing three times, ECL luminescent liquid was added dropwise, and a chemical imager was used to detect the chemiluminescence signals.

\section{Statistical analysis}

The measurement data in this study are presented as mean I standard error (SE). The ANOVA method was used to analyze the differences between the two groups, followed by Bonferroni post-hoc analysis or proper Student's $t$-test. A P 
A

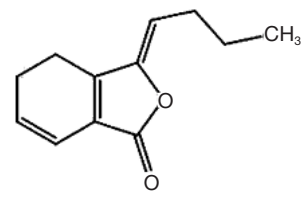

The chemical structure of Ligustilide

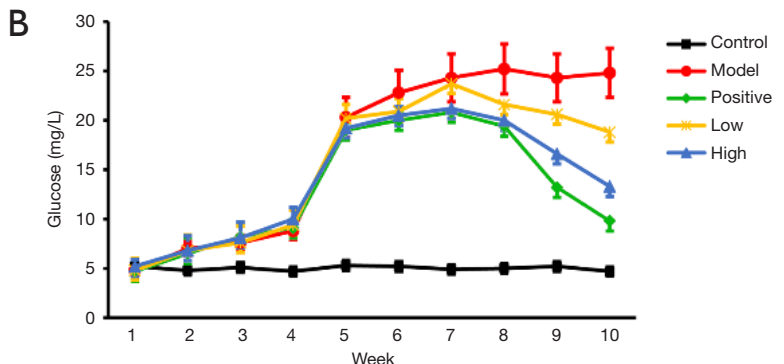

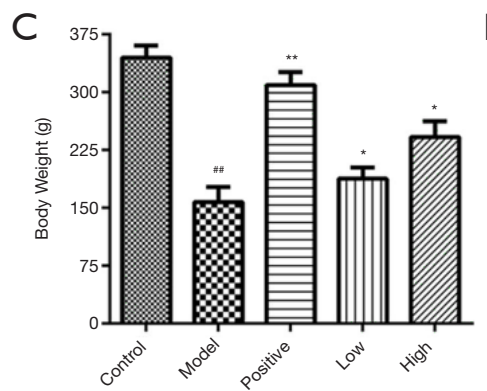
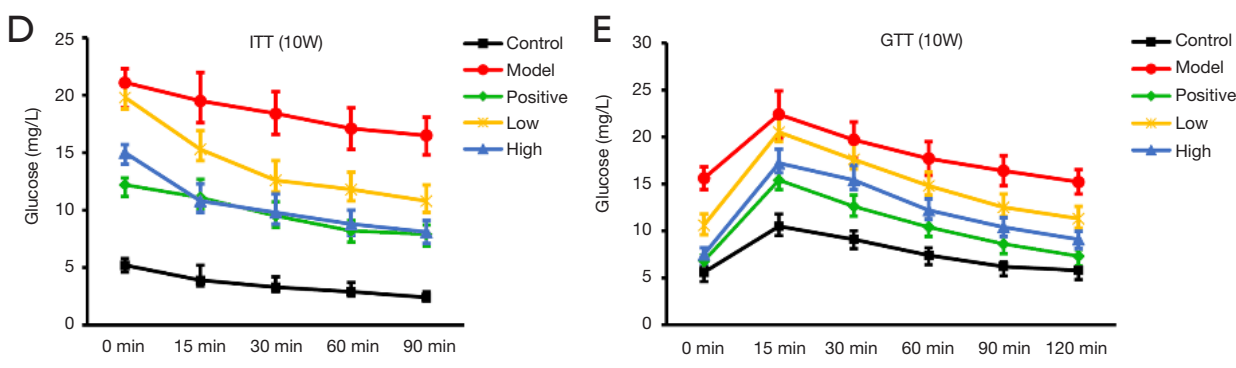

Figure 1 Effects of different treatments on body weight and blood glucose level. ${ }^{\#}, \mathrm{P}<0.01$ vs. control; ${ }^{*}, \mathrm{P}<0.05,{ }^{* *}, \mathrm{P}<0.01$ vs. model.

value of $<0.05$ was considered statistically significant.

\section{Results}

\section{Effect of LIG on body weight and insulin sensitivity in DN rat models}

The chemical structure of LIG is shown in Figure $1 \mathrm{~A}$. Other factors did not affect rats before modeling, and their weights were the same, as shown in Figure $1 B$. After STZ modeling, the weight of rats in the healthy group continued to increase steadily. Compared with the rats in the healthy group, DN rat models have slowly increased body weight and remarkably increased blood sugar levels. After the administration of Losartan potassium or different doses of LIG, the weight of the DN rat models increased slowly, and their blood sugar level was slightly decreased in a dosedependent manner (Figure 1B,C). GTT and ITT showed that $\mathrm{DN}$ rat models showed insulin resistance, which was markedly improved after treatment with losartan potassium or high- and low-dose LIG. It was found that the efficacy was more superior in the high-dose LIG group than in the low-dose LIG group and was comparable between the high-dose LIG group and the positive control group (Figure 1D,E). Thus, LIG could regulate the body weight and blood glucose level in DN rat models, and it was hypothesized LIG might protect kidneys in DN rat models.

\section{Effect of LIG on pathological changes and renal function in DN rat models}

Hematoxylin and eosin (HE) stains showed that, for rats in the healthy group, the structure of the glomerular filtration membrane (GBM) was intact, showing no thickening or wrinkling; also, the structures of glomerular capillaries and renal tubules were clear, and their lumens were not narrow. In DN rat models, numbers of glomeruli decreased, the capillary lumens shrank, along with inflammatory cell infiltration, increased mesangial cells and mesangial matrix, and glomerular and interstitial fibrosis in local areas. After the intervention with Losartan potassium or high- and low-dose LIG, the glomerular lesions were somehow improved, and the stenosis of the capillary lumen was reduced; also, the glomerular structure was not significantly damaged, and the proliferation of the mesangial matrix was relieved (Figure 2A).

The daily urinary output of rats in the healthy group was between 10-40 mL; meanwhile, the urine output of the $\mathrm{DN}$ rat models was between $40-150 \mathrm{~mL}$ and showed a gradually increasing trend, and the urine was thick and turbid and appeared foamy. After the intragastric administration, the urine output of $\mathrm{DN}$ rat models decreased, the urine viscosity and turbidity decreased, and the urine appeared less foamy. Compared with the healthy control group, the DN rat models had significantly higher levels of TC and TG; compared with DN rat models, the positive control group 
A

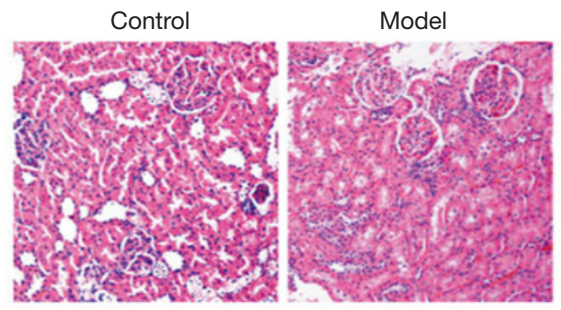

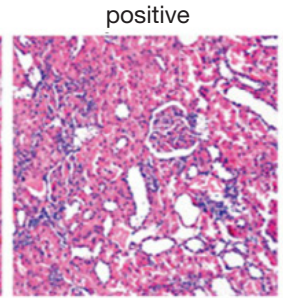

B
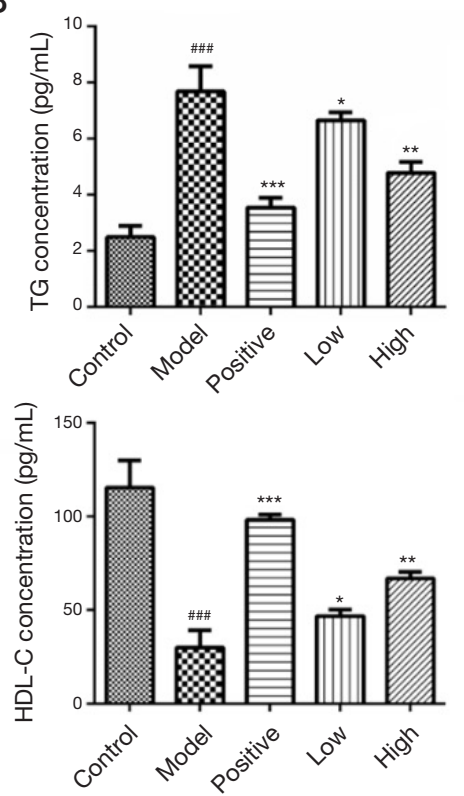
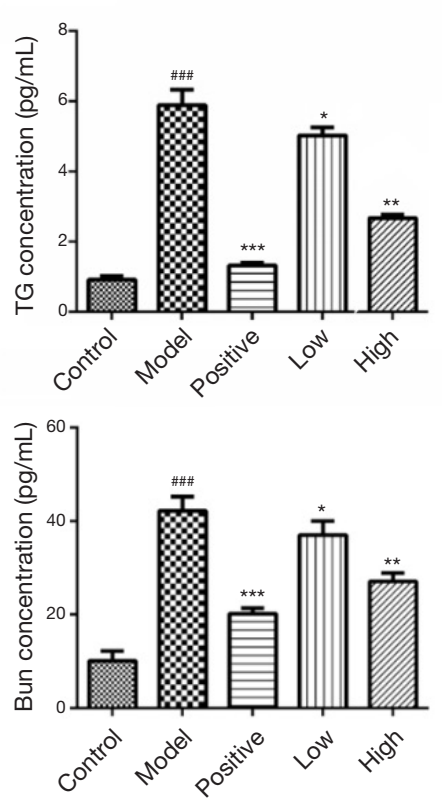

Low

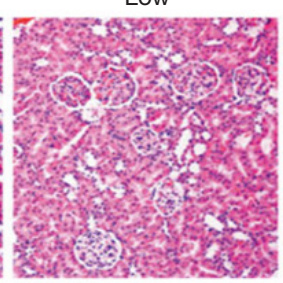

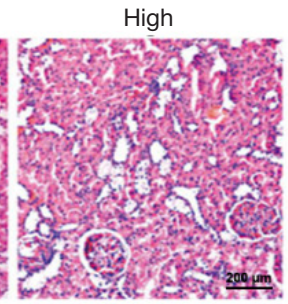
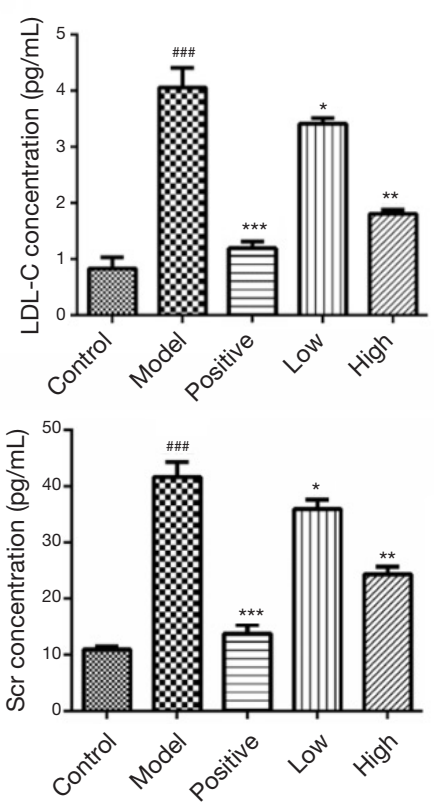

Figure 2 Effects of different treatments on renal histopathology and function in rats. ${ }^{* \# \#}, \mathrm{P}<0.001$ vs. control; ${ }^{*}, \mathrm{P}<0.05,{ }^{* *}, \mathrm{P}<0.01,{ }^{* * *}$, $\mathrm{P}<0.001$ vs. model.

and the high- and low-dose LIG groups had significantly decreased TC and TG levels, especially in the high-dose LIG group. Compared with the normal rats, the DN rat models had significantly increased LDL-C and decreased HDL-C levels; LDL-C levels significantly decreased, and HDL level significantly decreased after intervention with Losartan potassium or LIG. Compared with the normal rats, the DN rat models had significantly elevated BUN) and serum Scr; after intervention with Losartan potassium or LIG, both BUN and Scr decreased in the dosing groups (Figure 2B). Thus, LIG can remarkably improve the renal pathological changes and renal function in $\mathrm{DN}$ rat models, and the specific mechanism via which LIG protects renal function is explored in DN rat models.

\section{Effect of LIG on podocyte injury in DN rat models}

The glomerular podocytes can express nephrin, which takes part in supporting the integrity of the GBM.
Immunohistochemistry showed that the expression of nephrin in the kidney tissue of DN rat models decreased compared with that in the healthy group; after intervention with Losartan potassium or LIG, the expression of nephrin in the kidney tissue increased (Figure $3 A$ ), as also demonstrated by Western blotting (Figure 3B). Thus, LIG protected the kidney by alleviating podocyte injury.

\section{Effect of LIG on Sirtuin 1/nuclear factor-kappa B (SIRT1) NF- $\mathrm{kB}$ ) signaling pathway}

SIRT1 and NF- $\kappa \mathrm{B}$ are closely related to the pathogenesis of DN. Western blotting showed that compared with the healthy control group, the expression of SIRT1 protein in kidney tissue of DN rat models decreased, and the expression of p-NF- $\mathrm{kB}-\mathrm{p} 65$ protein increased. After the intervention with Losartan potassium or LIG, the abnormal expressions of SIRT1 and p-NF- $\mathrm{kB}-\mathrm{p} 65$ were regulated: the expression of SIRT1 protein increased, and the 
A Control Model Positive Low High

Nephrin
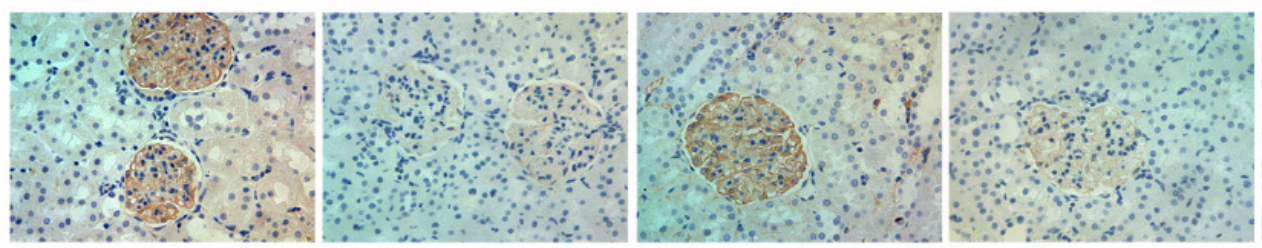

Nephrin

B

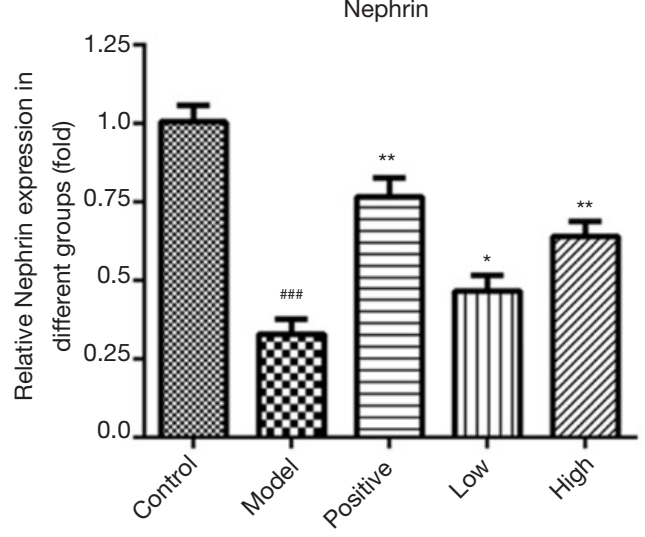

Figure 3 Effects of different treatments on nephrin expression in rat kidney tissue. ${ }^{\# \#}, \mathrm{P}<0.001 v s$. control; * $, \mathrm{P}<0.05,{ }^{* *}, \mathrm{P}<0.01$ vs. model.
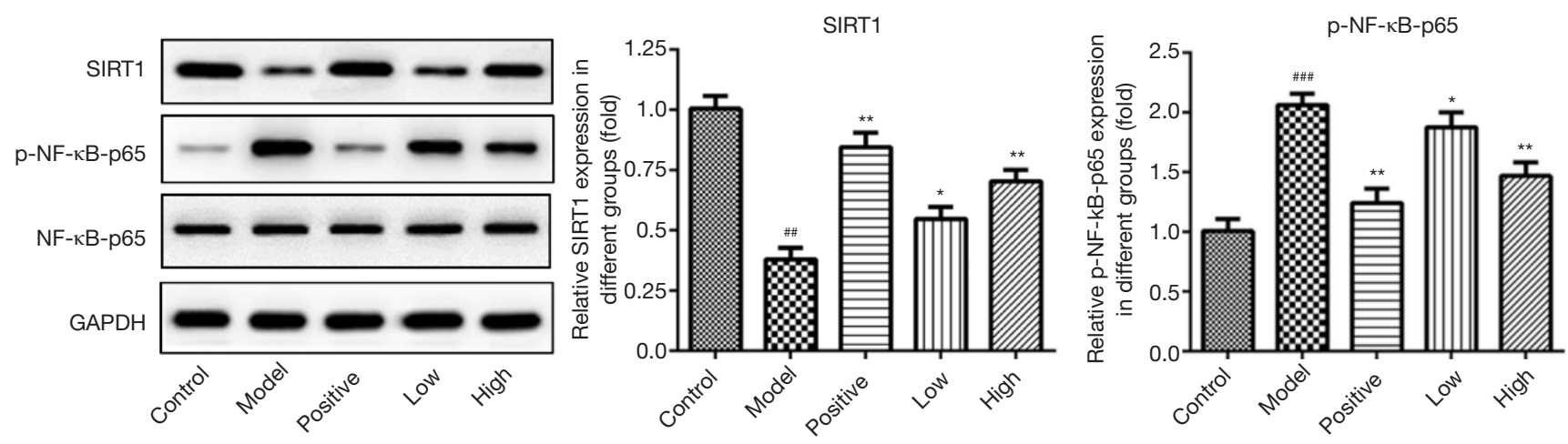

Figure 4 Effects of different treatments on the SIRT1/NF- $\kappa$ B signaling pathways. SIRT1/NF-Kb, Sirtuin 1/nuclear factor-kappa B signaling pathway. ${ }^{\#}, \mathrm{P}<0.01,{ }^{\# \#}, \mathrm{P}<0.001$ vs. control; * $, \mathrm{P}<0.05,{ }^{* *}, \mathrm{P}<0.01$ vs. model.

expression of p-NF- $\mathrm{kB}-\mathrm{p} 65$ protein decreased (Figure 4). Therefore, the SIRT1/NF- $\mathrm{kB}$ signaling pathways may be involved in the molecular mechanism via which LIG exerts its pharmacological effects on DN, although the specific pharmacological mechanism needs to be further verified.

\section{Discussion}

$\mathrm{DN}$ is a serious microvascular complication of diabetes. Studies have shown that the incidence of DN is rising in developed countries (13). DN often develops into end-stage renal disease (ESRD) (2) and thus is exceptionally harmful. Compared with other kidney diseases, DN has complex metabolic disorders, which make the treatment more challenging. Therefore, R\&D of drugs that can effectively prevent and treat $\mathrm{DN}$ is of considerable significance to clinically treat or delay the course of DN. In our current study, we established DN rat models using STZ combined with a high-fat diet and, for the first time, demonstrated the therapeutic effect of LIG on DN. Consistent with the findings of a previous study (14), our study showed that DN rats had elevated blood glucose levels, glomerular atrophy, 
mesangial and stromal proliferation, inflammatory cell infiltration, and proteinuria.

In recent years, traditional Chinese medicine (TCM) therapies for DN, especially the application of the active ingredients of TCM for the prevention of $\mathrm{DN}$, have produced breakthroughs. Fan et al. found that curcumin could improve adriamycin-induced nephrotic syndrome in rats by inhibiting the NF- $\mathrm{B}$ pathway (15). Li et al. proved resveratrol alleviated early podocyte injury by improving lipid metabolism and insulin sensitivity and inhibited inflammatory responses in obese rats induced by an ovariectomy with an HFD (16). LIG accounts for the main pharmaceutical activities in traditional Chinese drugs, including Radix Angelicae Sinensis, Rhizoma Ligustici Cbuanxiong, and other plants in the family Apiaceae. With its anti-inflammatory and antioxidant activities, LIG can lower blood sugar levels and improve the pathological changes of the kidneys in DN rats. In our current study, it reduced the TC, TG, LDL-C, BUN, and Scr levels increased the HDL-C level and alleviated podocyte injury in DN. It showed a protective effect on the renal function of DN rats. Thus, LIG can alleviate renal dysfunction in $\mathrm{DN}$ rats and has a specific therapeutic effect on DN.

The early clinical symptom of $\mathrm{DN}$ is microalbuminuria, associated with podocyte injury. Nephrin, a transmembrane protein expressed in glomerular podocytes, takes part in supporting the integrity of the GBM. Podocyte injury occurs in the early stages of DN. When the binding of nephrin protein on the surface of podocytes with skeletal protein is weakened, nephrin sheds off from the podocytes and is excreted into the urine. Therefore, podocyte dysfunction is an essential factor in the pathogenesis of DN. In our current study, the expression of nephrin decreased in the kidney tissue of DN rats and increased after intervention with LIG, suggesting LIG has a protective effect on the function of podocytes in $\mathrm{DN}$ and reduces the high glucoseinduced podocyte injury.

Lei et al. found that LIG could effectively inhibit the expression of $\mathrm{NF}-\kappa \mathrm{B}$, thereby exerting its antiinflammatory effect and improving atherosclerosis (17). Li et al. demonstrated that LIG could reduce IL-1 $\beta$ induced inflammation in chondrocytes by inhibiting the activation of NF- $\kappa \mathrm{B}$ and had a specific therapeutic effect on osteoarthritis (18). Also, the expression of NF- $\kappa B$ and the acetylation level of signal transducer and activator of transcription 3 (STAT3) increased in diabetic kidneys; when SIRT1 expression was up-regulated, the expression of NF-кB-p65 and the acetylation level of STAT3 were inhibited (19). Many studies have shown SIRT1 plays a vital role in the pathogenesis of $\mathrm{DN}$, and some synthetic drugs and natural compounds can up-regulate the expression and activity of SIRT1, thereby protecting kidney function (20); however, no literature has investigated the effect of LIG on SIRT1 in DN. In our current study, we found that LIG could inhibit the abnormal activation of NF- $\kappa \mathrm{B}$ in $\mathrm{DN}$ rats; also, it up-regulated the expression of SIRT1 and thus restored its regulatory effect on the NF- $\kappa \mathrm{B}$ pathway. The effect of LIG on the SIRT1/NF- $\kappa$ B signaling pathways may also explain the therapeutic effect of LIG on DN.

In summary, our research has proved that LIG can reduce the symptoms of renal dysfunction in DN rats. LIG may exert its therapeutic effect by regulating the SIRT1/ $\mathrm{NF}-\mathrm{\kappa B}$ signaling pathways and thus alleviating the podocyte injury under a high glucose environment. However, since the metabolism is complex in DN rats and may involve many pathways, the specific targets of LIG still call for further investigations. What's more, LIG is a traditional Chinese drug, the side effects have not been well identified, therefore, more work is needed in this field.

\section{Acknowledgments}

Funding: This study is supported by the Youth Project of Nantong Health Committee of Jiangsu Province (QA2019001) and the General Project of Nantong Health Committee of Jiangsu Province (MB2019011).

\section{Footnote}

Reporting Checklist: The authors have completed the ARRIVE reporting checklist. Available at http://dx.doi. org/10.21037/atm-20-5811

Data Sharing Statement: Available at http://dx.doi. org/10.21037/atm-20-5811

Conflicts of Interest: All authors have completed the ICMJE uniform disclosure form (available at http://dx.doi. org/10.21037/atm-20-5811). The authors have no conflicts of interest to declare.

Ethical Statement: The authors are accountable for all aspects of the work in ensuring that questions related to the accuracy or integrity of any part of the work are appropriately investigated and resolved. The office of the Laboratory Animal Welfare and the Ethics Committee at 
Nantong University approved the study. The breeding of these animals followed the requirements in the Regulations of the People's Republic of China on the Management of Laboratory Animals and the Measures for the Quality Management of Laboratory Animals (Animal Ethics Review Number: No. S20191021-003).

Open Access Statement: This is an Open Access article distributed in accordance with the Creative Commons Attribution-NonCommercial-NoDerivs 4.0 International License (CC BY-NC-ND 4.0), which permits the noncommercial replication and distribution of the article with the strict proviso that no changes or edits are made and the original work is properly cited (including links to both the formal publication through the relevant DOI and the license). See: https://creativecommons.org/licenses/by-nc-nd/4.0/.

\section{References}

1. Li SY, Huang PH, Tarng DC, et al. Four-and-aHalf LIM Domains Protein 2 Is a Coactivator of Wnt Signaling in Diabetic Kidney Disease. J Am Soc Nephrol 2015;26:3072-84.

2. Soler MJ, Batlle D. Single-cell RNA profiling of glomerular cells in diabetic kidney: a step forward for understanding diabetic nephropathy. Ann Transl Med 2019; 7:S340.

3. Ziyadeh FN, Wolf G. Pathogenesis of the podocytopathy and proteinuria in diabetic glomerulopathy. Curr Diabetes Rev 2008;4:39-45.

4. Mandache E, Penescu M. Nanostructural features of diabetic podocytopathy. Rom J Morphol Embryol 2012;53:23-27.

5. Dai HY, Zheng M, Tang RN, et al. Effects of angiotensin receptor blocker on phenotypic alterations of podocytes in early diabetic nephropathy. Am J Med Sci 2011;341:207-14.

6. Lv Z, Hu M, Zhen J, et al. Rac1/PAK1 signaling promotes epithelial-mesenchymal transition of podocytes in vitro via triggering $\beta$-catenin transcriptional activity under high glucose conditions. Int J Biochem Cell Biol 2013;45:255-64.

7. Bhatti AB, Usman M. Drug Targets for Oxidative Podocyte Injury in Diabetic Nephropathy. Cureus 2015;7:e393.

8. Zhou Y, Ming J, Li Y, et al. Ligustilide attenuates nitric oxide-induced apoptosis in rat chondrocytes and cartilage degradation via inhibiting JNK and p38 MAPK pathways. J Cell Mol Med 2019;23:3357-68.

9. Choi ES, Yoon JJ, Han BH, et al. Ligustilide attenuates vascular inflammation and activates $\mathrm{Nrf} 2 / \mathrm{HO}-1$ induction and, NO synthesis in HUVECs. Phytomedicine 2018;38:12-23.

10. Donkor PO, Chen Y, Ding L, et al. Locally and traditionally used Ligusticum species - A review of their phytochemistry, pharmacology and pharmacokinetics. J Ethnopharmacol 2016;194:530-48.

11. Barzegar-Fallah N, Baery N, Soleymankhani M, et al. Kelussia odoratissima Mozaff attenuates thromboembolic brain injury, possibly due to its Z-ligustilide content. Brain Inj 2016;30:926-32.

12. Chi K, Fu RH, Huang YC, et al. Therapeutic Effect of Ligustilide-Stimulated Adipose-Derived Stem Cells in a Mouse Thromboembolic Stroke Model. Cell Transplant 2016;25:899-912.

13. Feng Y, Weng H, Ling L, et al. Modulating the gut microbiota and inflammation is involved in the effect of Bupleurum polysaccharides against diabetic nephropathy in mice. Int J Biol Macromol 2019;132:1001-11.

14. Xie S, Ge F, Yao Y, et al. The aqueous extract of Lycopus lucidus Turcz exerts protective effects on podocytes injury of diabetic nephropathy via inhibiting TGF- $\beta 1$ signal pathway. Am J Transl Res 2019;11:5689-702.

15. Fan HY, Wang XK, Li X, et al. Curcumin, as a pleiotropic agent, improves doxorubicin-induced nephrotic syndrome in rats. J Ethnopharmacol 2020;250:112502.

16. Li B, Xiao X, Miao Y, et al. Resveratrol alleviates obesityassociated podocyte injury in ovariectomized obese rats. Exp Ther Med 2020;19:123-30.

17. Lei W, Deng YF, Hu XY, et al. Phthalides, senkyunolide A and ligustilide, show immunomodulatory effect in improving atherosclerosis, through inhibiting AP-1 and NF-kappaB expression. Biomed Pharmacother 2019;117:109074.

18. Li X, Wu D, Hu Z, et al. The Protective Effect of Ligustilide in Osteoarthritis:An in Vitro and in Vivo Study. Cell Physiol Biochem 2018;48:2583-95.

19. Liu R, Zhong Y, Li X, et al. Role of transcription factor acetylation in diabetic kidney disease. Diabetes 2014;63:2440-53.

20. Wang W, Sun W, Cheng Y, et al. Role of sirtuin-1 in diabetic nephropathy. J Mol Med (Berl) 2019;97:291-309.

Cite this article as: $\mathrm{Xu} \mathrm{F,} \mathrm{Fang} \mathrm{X,} \mathrm{Ye} \mathrm{Z,} \mathrm{Tao} \mathrm{S,} \mathrm{Liu} \mathrm{W,} \mathrm{Su} \mathrm{J,}$ Wang X. Ligustilide alleviates podocyte injury via suppressing the SIRT1/NF- $\kappa \mathrm{B}$ signaling pathways in rats with diabetic nephropathy. Ann Transl Med 2020;8(18):1154. doi: 10.21037/ atm-20-5811 